\title{
Electron transport behavior of quinoidal heteroacene-based junctions: Effective electron-transport pathways and quantum interference
}

\author{
Na Cheng ${ }^{1,3 *}$, Feng Chen ${ }^{1}$, Colm Durkan ${ }^{2}$, Nan Wang ${ }^{2}$, Yuanyuan $\mathrm{He}^{1}$, Jianwei Zhao ${ }^{1 *}$ \\ ${ }^{I}$ College of Material and Textile Engineering, China-Australia Institute for Advanced Materials and \\ Manufacturing, Jiaxing University, Jiaxing 314001 \\ ${ }^{2}$ Nanoscience Centre, University of Cambridge, Cambridge, CB3 OFF, UK \\ ${ }^{3}$ Department of Polymer Science \& Engineering, and Department of Chemistry, Zhejiang University, Hangzhou \\ 310027, China
}

\begin{abstract}
The electron transport behavior through a series of molecular junctions composed of tetracene (TC) and S/O substituted-TC (S/O-TC) has been studied using density functional theory (DFT) combined with the non-equilibrium Green's function (NEGF) method. The unique transport behavior has been interpreted using correlated quantum interference and electron transport pathway models. In the TC system, two dominant electron transfer channels exist as demonstrated by detailed transmission pathway analysis. In the substituted S/O-TC systems, the electron transport behavior is regulated through either constructive or destructive quantum interference due to the existence of additional $p$-electrons, leading to a significant diversity of current-voltage curves. Compared to the $\mathrm{TC}$ molecule in the bias region from 0 to $1.0 \mathrm{~V}$, an $\alpha$-connected molecular junction exhibits greater current, whereas a $\beta$-connected molecular junction shows a smaller current. The substitution with $\mathrm{O}$ and $\mathrm{S}$ atoms shows a minor effect on the conductance of the molecular junctions. In order to clarify the role of heteroatoms, a series of artificial models designed by removing specific sulfur and carbon atoms in $\alpha$-S-TC have been investigated in detail. The results have demonstrated that only the $\mathrm{S}$ heteroatom on one side of the molecule contributes to the junction conductivity through constructive quantum interference. It has been also observed that current exchange occurs between the two electron transfer channels.
\end{abstract}

Keywords: Electron transport, Quantum interference, Effective electron-transport pathway, Heteroacene 
*To whom all correspondence should be addressed.

Email: nacheng@mail.zjxu.edu.cn and jwzhao@mail.zjxu.edu.cn 


\section{Introduction}

The miniaturization of silicon-based microelectronic devices is approaching fundamental physical limits, therefore, single-molecule electronic devices have gained great attention over the past few decades ${ }^{1-7}$. Molecular electronic devices include switches $^{8,9}$, rectifiers ${ }^{10,11}$, negative differential resistance (NDR) ${ }^{12,13}$ and memories ${ }^{14}$, but conducting molecular wires are of particular importance as they are the basis of the other functionalities. A great deal of experimental and computational work has been

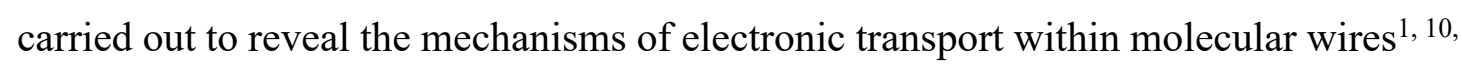
13, 15-17. These investigations have found that there are many factors, such as electrode material $^{1,18-20}$, interfacial structure ${ }^{21,22}$, anchoring groups ${ }^{23,24}$, as well as the molecule itself $1,16,17,25$ playing a role in molecular electron transport. The effect of molecular structure on the transport behavior has been especially concerned by chemists, since the delicate design of molecular structure bring diverse electron transport behaviors. For instance, molecules with a similar structure could display similar current-voltage relationships ${ }^{26}$, and the conductance can be altered by several orders of magnitude when molecules have different connections of anchoring groups ${ }^{27,28}$. The effective electrontransport pathway and quantum interference have been successfully used to explain these observed phenomena.

Quantum interference (QI) can occur when electrons tunnel through a molecule via more than one pathway, in which case the electron wavefunctions corresponding to the different pathways may be in-phase or out-of-phase with each other. Sautet and Joachim ${ }^{29}$ have studied interference of electrons transmitting through benzene which is embedded in a polyacetylene chain. It was deduced that QI significantly alters the electronic transmission through benzene in the meta and ortho cases, but not the para case. Baer and Neuhauser have investigated the application of interference for controlling the conductance of simple molecular devices such as polyacetylene loops of various lengths and a series of differently positioned polycyclic aromatic rings. ${ }^{30-34}$ By studying the junction conductance of unsaturated carbon chains ${ }^{35}$, pyridine ${ }^{36}$, anthraquinone ${ }^{37}$, and azulene ${ }^{38,39}$, the results demonstrated that the QI can be controlled by chemical design due to the intimate relationship between QI and the shape and energy of the molecular orbitals involved. The QI effect can thus be used to explain electron transport in molecular junctions. However, it is not as intuitive as the effective electron-transport pathway method. 
The electron-transport pathway refers to the local space with the highest probability of electron transfer, which is based on the concept of valence bond theory. It can be more intuitively represented as the relationship between the transfer behavior and the molecular structure. In our previous work, we have shown that an electron transfer channel exists in organic molecules embedded between two gold electrodes, and that the junction conductance is strongly dependent on the effective electron-transport pathway. ${ }^{40-43}$

Among various electronic materials in existence, oligoacenes and their derivatives are regarded as prominent organic semiconductors. These molecules have attracted tremendous interest due to their unique physical properties and potential applications in organic electronics and energy storage. ${ }^{44-46}$ Compared with these conventional aromatic molecules, quinoidal cyclic compounds display unique optical, electronic and magnetic properties ${ }^{44-46}$ because of their intrinsic open- or closed-shell diradical character. Many heterocyclic quinoidal compounds containing $\mathrm{N}, \mathrm{O}, \mathrm{Si}$ or $\mathrm{S}$ heteroatoms in the quinoidal unit, have been synthesized and their structural and electronic properties have been studied. ${ }^{44}$ These studies found that the neutral heteroacenes have a quinoidal-conjugated structure in the ground state, and the diradical character of these compounds increases with extension of the central quinoidal sextet-cyclic unit. $^{46}$ Compared to pentacene, 5,12-dithiapentacene has increased HOMO energy levels and more efficient electron transfer performance, due to the electron-donating nature of the thioether linkage in the backbone. ${ }^{45}$ In spite of the potential applications for organic electronics based on the electronic properties and transport behavior of heteracenes, they have rarely been reported on experimentally. In the present work, we have used tetracene (TC) and heteroacenes containing two sulfur or oxygen atom as computational models, using density functional theory combined with the nonequilibrium Green's function method (DFT-NEGF), to investigate the transport behavior of heteroacene-based junctions involving different terminal connections, and to illuminate the influence of QI and electron-transport pathway on molecular conductance properties by incorporation of heteroatoms.

\section{Methodology}

Incorporation of $\mathrm{S}$ or $\mathrm{O}$ heteroatoms into the acene framework will result in quinoidal conjugation ${ }^{47}$. The quinoidal conjugation could enhance electrical 
characteristics of electronic materials. Scheme 1a displays the structures of the studied tetracene and quinoidal heteroacenes, namely O-substituted TC (O-TC) and Ssubstituted TC (S-TC). In this work, by using these molecules as study models, we investigate the effect of heteroatom substitution and different terminal connections on the molecular electron transport behavior. Furthermore, in order to study the influence of the heteroatoms and transport pathways, five artificial models called as $\alpha$-S-TC-1, $\alpha$ S-TC-2, $\alpha$-S-TC-3, $\alpha$-S-TC-4 and $\alpha$-S-TC-5 (in Scheme 1b), are designed by removing specific sulfur or carbon atoms in the $\alpha$-S-TC molecule. The geometries of the model molecules were optimized using density functional theory (DFT) at the B3LYP/Lanl2DZ level, as implemented in the Gaussian 09 package $^{47}$. This functional has been used widely and proven to be accurate for optimizing the geometry of organic molecules. ${ }^{48,49}$ For each model, the molecule was embedded at the hollow site of two small gold clusters, each of which is an equilateral triangle of Au atoms, with an Au$\mathrm{Au}$ bond length of $2.88 \AA .^{40-43}$ It can provide a good electrical contact between the organic molecule and the metal electrode. The relative positions of $\mathrm{Au}$ atoms were frozen in each triangle, but the distances between the two clusters were relaxed during geometry optimization.

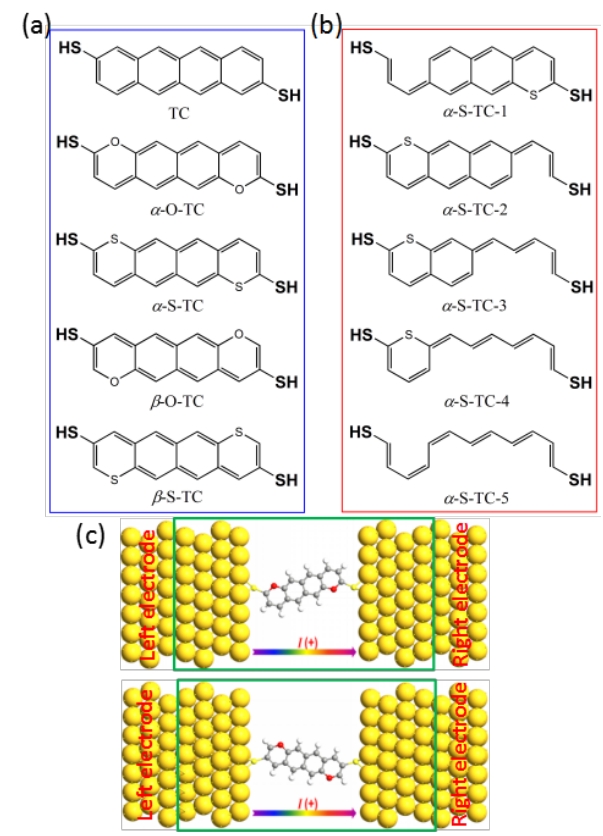

Scheme 1 (a) The molecular structures of tetracene (TC), $\alpha$ - and $\beta$-connected heteroacenes; (b) the structures of five artificial models, which are designed by removing certain sulfur or carbon atoms; (c) Schematic representation of the molecular junction, while $4 \times 4 \mathrm{Au}$ atoms were used to simulate the electrodes. 
Scheme 1c illustrates the representative model of the molecular junction with $\mathrm{Au} / \mathrm{molecule} / \mathrm{Au}$ structure, in which the heteroacene with dithiolate groups are sandwiched between two gold electrodes. The thiolate end group is adopted extensively in experimental and theoretical studies of molecular devices. ${ }^{1,21,50,51}$ The optimized geometries and transport behaviors of molecular junctions were studied by DFT-NEGF. The $(4 \times 4)$ unit cells with periodic boundary conditions of the Au (111) surface were used as the metallic electrode. The generalized gradient approximation (GGA-PBE) is used to calculate the electron exchange and correlation. ${ }^{[15]}$ The Double zeta polarization (DZP) basis set was employed for $\mathrm{C}, \mathrm{H}, \mathrm{O}$ and $\mathrm{S}$ atoms, and the single zeta polarization (SZP) basis set for the Au atom. In the optimization, the central organic molecule was relaxed completely, while the coordinates of the electrode atoms were frozen to take account of the known strong interaction among $\mathrm{Au}$ atoms. The transport properties, including the current-voltage (I-V) characteristic, density of states (DOS) and transmission spectra of the molecular junctions were calculated on the basis of the above optimization results. The geometry optimizations and electron transport computations of the molecular junctions were carried out using the Atomistix Toolkit (ATK) package ${ }^{52}$.

\section{Results and discussion}

\section{Electron transfer behavior of the tetracene and quinoidal heteroacenes}

The strong correlation between electronic structure and electrical properties is an important feature in organic $\pi$-conjugated materials. The electronic structures of molecules can be effectively adjusted by doping with heteroatoms. ${ }^{1,20,44-46,53-56}$ To study the impact of heteroatoms on the electronic structure nature of TC and substituted TC, we compared the spatial distribution of the highest occupied molecular orbital (HOMO) and the lowest unoccupied molecular orbital (LUMO) for all samples in Figure 1a. For TC, both HOMO and LUMO are uniformly distributed over the molecule chain, indicating the well-conjugated and therefore delocalized electronic structure. Compared to carbon, sulfur and oxygen atoms have two more valence electrons that may take part in the conjugation together with other carbon atoms along the backbone. $\mathrm{O} / \mathrm{S}$-substituted TC has the quinoidal $\pi$-conjugation structure, which is different to the diene conjugation structure of TC. ${ }^{44-46}$ As oxygen and sulfur atoms adopt $s p^{2}$-hydridized state in $\mathrm{S} / \mathrm{O}-\mathrm{TC}$, one of the lone pairs of electrons is delocalized within a sextet ring. ${ }^{57}$, 
${ }^{58}$ Therefore, the molecular orbitals are significantly different from those within the TC molecule. As can be seen from Figure 1a, the HOMO and LUMO of O- and Ssubstituted TC displayed a similar spatial distribution implying that these models may have similar transport behavior. However, the frontier molecular orbitals of the $\alpha$ - and $\beta$-connected $\mathrm{O} / \mathrm{S}$-TC showed significant differences. The HOMO and LUMO orbitals of $\alpha$-connected TC are spread over the whole molecular chain, indicating that molecules with $\alpha$-connection are well coupled to the gold electrodes. Compared to $\alpha$ connection, the distribution of HOMO and LUMO does not extend to the anchoring groups of $\beta$-connected models. Thus, $\alpha$-connection is expected to result in a junction with higher conductance than $\beta$-connection.

As well as having different molecular orbital distributions, the energy levels of $\mathrm{O} / \mathrm{S}$ TC and TC display significant differences. TC has the smallest HOMO value and the largest LUMO value, while the S/O-TC have increased HOMO and decreased LUMO energy levels. That is, the heteroatom-substitution leads to decreased HOMO-LUMO gaps of heteroacenes. Besides, the molecular orbital levels are sensitive to the applied bias as shown in Figure 1b, from which we can see the variation of HOMO and LUMO as a function of bias. With an increase of bias, the HOMO gradually enters into the bias window, but the LUMO always stays out of it. At higher bias voltages, the HOMO enters into the bias windows contributing to the current. However, under low and moderate bias, the HOMO of TC is always kept out of the bias window, while the HOMO of heteroacences enters into the bias window contributing to the current. This implies that TC and O/S-TC will display diverse electron-transport behaviors.
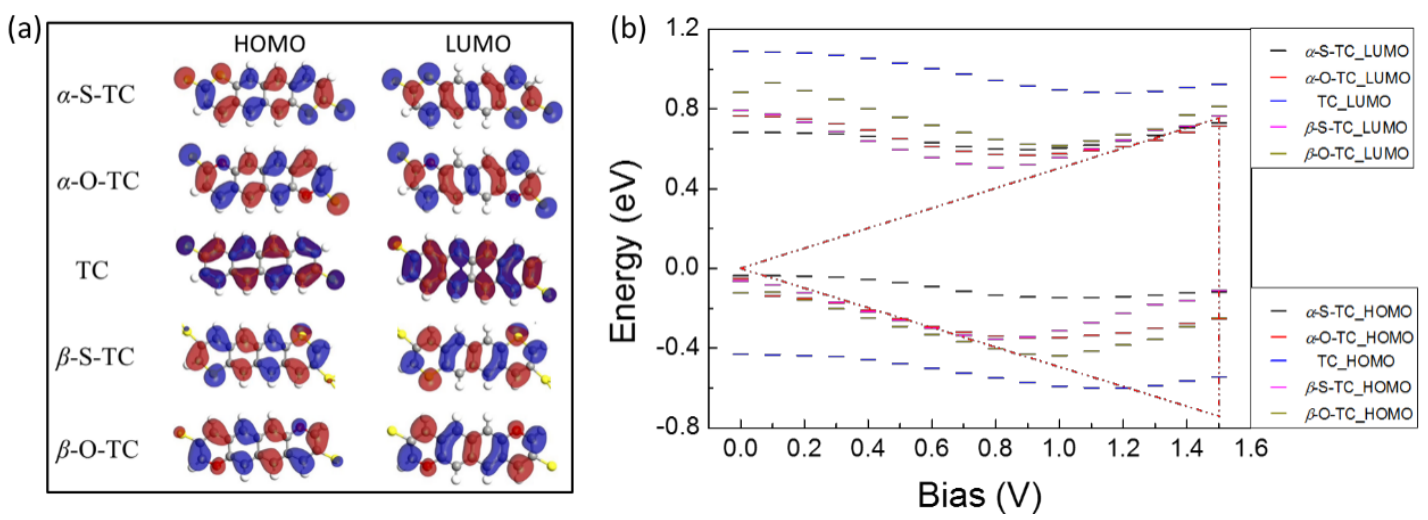

Figure 1. (a) The spatial distribution of HOMO and LUMO at the zero bias; (b) the HOMO and LUMO energy levels with the bias of all molecules.

It has been shown that the incorporation of two heteroatoms can indeed modify 
local electronic states of the electron transfer pathway, and modulate the transport behavior. Figure 2 shows the calculated $I-V$ characteristics of all models. At low bias $(<0.3 \mathrm{~V})$, the current through TC, $\alpha$-O-TC and $\alpha$-S-TC increases almost linearly with an increase of bias. However, at higher bias, all models show nonlinear features, similar to the experimental observations of fused conjugated molecules. ${ }^{59}$ The calculated $I-V$ characteristic of TC is similar to that observed experimentally by Kim et al. ${ }^{59}$ Taking the TC molecular junction as a reference, there are there clear categories of junction conductivity as shown in Figure 2. The current through $\alpha$-connected heteroacenes is largest, followed by TC, and finally $\beta$-connected heteroacenes have the smallest current. For example, at a bias voltage of $1.0 \mathrm{~V}$, the current through $\alpha$-connected S/O-TC is approx. $30 \mu \mathrm{A}$, which is six times larger than that $(\sim 5 \mu \mathrm{A})$ of $\beta$-connected S/O-TC. This implies that electron transport through $\alpha$-connected molecules is significantly more efficient. Somewhat surprisingly, there is no significant difference between the Osubstitution and S-substitution, implying that the modulation of the junction conductivity may be strongly dependent on the outermost electron structure of these heteroatoms.

The origin of the diversity of the electron transfer behavior of this series of molecules can be seen by considering the Hückel model and DFT calculations ${ }^{60}$. In TC and $\alpha$-connected $\mathrm{S} / \mathrm{O}$-TC systems, electrons follow the effective electron-transport pathway, and destructive QI is absent. However, destructive quantum interference is present in the electron transfer behavior of the $\beta$-connected system, resulting in the smaller conductivity. According to a previous study, the TC system has two electrontransport pathways with the same phase, leading to so constructive QI. ${ }^{26}$ In the present study, there are two electron-transport pathways in TC and the $\alpha$-connected system, but their currents display different characteristics. This can be attributed to the properties such as density of states (DOS) and transmission, of these molecular junctions. 


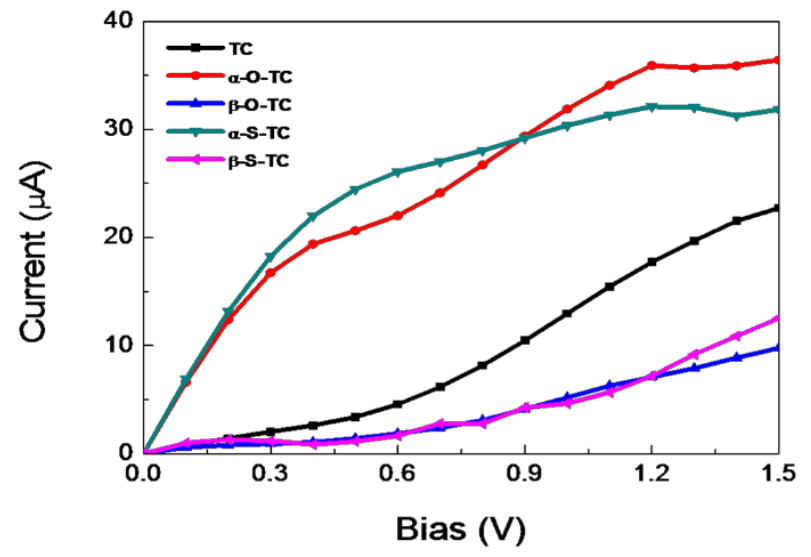

Figure 2. The plots of $I-V$ curves of TC, O-TC and S-TC systems.

In addition to the molecular orbital distribution, another property that may reflect the static feature of the system as a whole is the density of states (DOS). Since the molecule is sandwiched between two metallic electrodes in the device, the effect from the electrode cannot be simply ignored. As the contribution of the electrodes to the DOS is much larger than that of the molecules, the differences in molecular contributions to the DOS are obscured. Therefore, in order to study the effect of the molecule on the DOS, the projected density of states (PDOS) involving the influence of the two metallic electrodes, which is the DOS projected onto the molecule itself, should be taken into account. ${ }^{61,62}$ Figure 3 a presents the projected density of states (PDOS) of all molecules. It is most relevant to analyze the PDOS around the Fermi level. For TC, the closest peak appears at $-0.4 \mathrm{eV}$ with the next at $1.12 \mathrm{eV}$. They contribute less to the PDOS value at the Fermi level. This feature of the PDOS indicates that the conductance may be dominated by hole transport in TC. When TC is substituted, these peaks move toward the Fermi level. In particular, the peak positions are located at about -0.08 and $0.0 \mathrm{eV}$ for $\beta$-connection and $\alpha$-connection, respectively. These peaks are primarily due to the HOMO levels of the heteroacenes. This implies that the conductance mechanism is electron transport for $\mathrm{O} / \mathrm{S}$ - TC moelcules. The height of the peaks depends more strongly on the type of connection than on the type of substitution (S Vs O).

In order to clarify the electron transfer mechanism of quinoidal heteroacene, we further investigated the transmission spectrum of the junction as shown in Figure $3 \mathrm{~b}$. The transmission coefficient is involved in the Landauer-Büttiker equation ${ }^{63,64}$ that can 
reasonably describe the junction current,

$$
I=\frac{2 e}{h} \int\left[F_{L}\left(\varepsilon, V_{b}\right)-F_{R}\left(\varepsilon, V_{b}\right)\right] T\left(\varepsilon, V_{b}\right) d \varepsilon
$$

where $F_{L}\left(\varepsilon, V_{b}\right)$ and $F_{R}\left(\varepsilon, V_{b}\right)$ are the Fermi-Dirac distribution functions for the left and right metallic electrodes at energy level $\varepsilon$ with a given bias voltage $V_{b} . T\left(\varepsilon, V_{b}\right)$ is the transmission coefficient, which is a function of the energy level $\varepsilon$ and bias $V_{b}$. From Equation (1), the transmission contributes to the total current in the junction, especially the transmission peak close to the Fermi level. Transmission spectra can reveal the details of the electron transport, which help us to understand the influence of the heteroatoms. In general, the transmission curve shows similar features to the PDOS. For instance, the transmission peak of TC below the Fermi level appears at $-0.4 \mathrm{eV}$ exactly the same as that of the PDOS. In addition, the peak height is about 0.9, indicating a good conductivity of the sample, which is also similar to other conductive molecular wires. ${ }^{40 a}, 59$ When TC is substituted by $\mathrm{O}$ and $\mathrm{S}$ atoms, the transmission peaks shift to the Fermi level as do the PDOS peaks. Compared to the substitution effect, the connection position of the anchoring group dominates in the overall characteristics of the transmission. When the substituted molecule is connected to the electrodes with the $\beta$-position, the peak becomes small and narrow. However, a positive shift of the peak position compensates this change. Therefore, the zero-bias conductivity of TC and $\beta$ connected models are very close. In the case of $\alpha$-connection, the transmission peak does not only move towards the Fermi level, but also possesses a large transmission value close to 1 . As a result, the $\alpha$-connected heteroacenes will have much greater zerobias conductivity than TC and heteroacenes with $\beta$-connection.
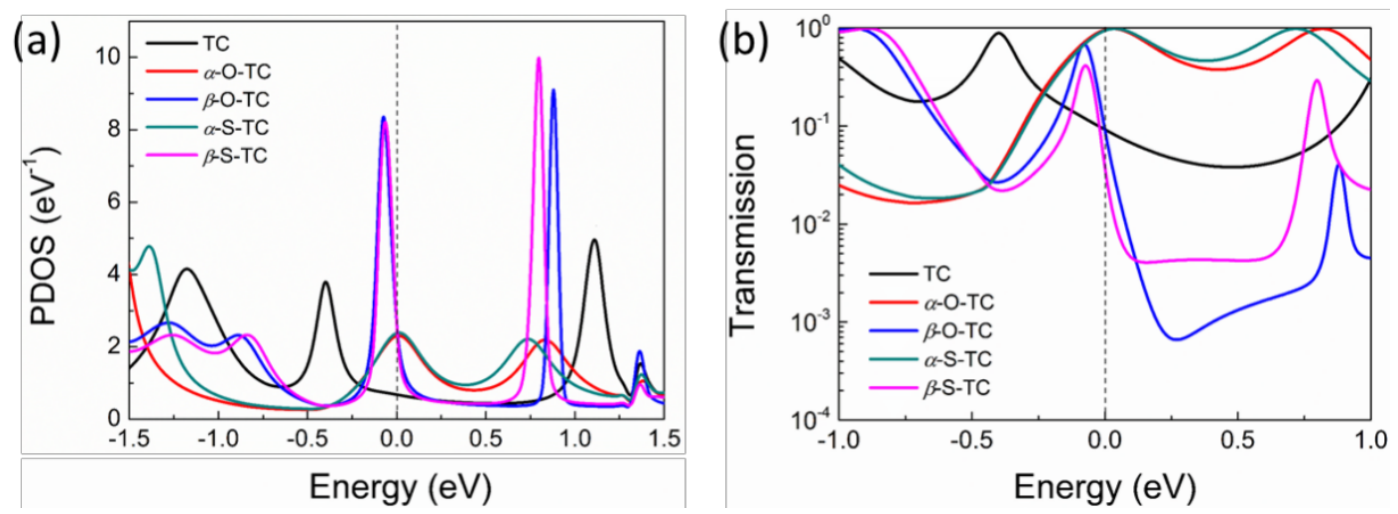

Figure 3. (a) The density of states projected on the molecular region (PDOS), (b) the transmission 
spectra of TC, $\alpha$-connected and $\beta$-connected quinodial heteroacenes.

The transmission Pathway is an analysis option which splits the transmission coefficient into local bond contributions ${ }^{65}$. Drawing the spatial distribution of the transmission probability can aid our understanding of the terminal connection and heteroatom-substitution effect from a microscopic viewpoint. Although electrons at the junction can transfer through any position around the molecule, the probability of emergence is not the same for all paths due to differences in the local chemical environment. The consecutive positions with larger transmission probability provide an intuitive explanation of the electron-transfer pathway. Figure 4 shows the transmission pathway at the Fermi level of all five molecules. The transmission pathway is represented by the blue arrows distributed over the molecular structure with a threshold of 0.2 . Without any substitution, the electron flows through each of two parallel routes, between which the weak current exchanges establish a connection. Since each parallel route is relatively efficient at electron transfer, TC behaves as a good conductive molecular wire. However, the current exchange between the two parallel routes is less efficient. It is thus of interest to see how the heteroatom substitution modulates the junction current. In the $\alpha$-substitution, the heteroatoms facilitate the electron injection to the Route 2 as shown in Figure 4 due to the current exchange between two electrontransport pathways. Unlike TC, the current exchange between Route 1 and Route 2 appears to be more efficient, showing that Route 1 becomes wider and Route 2 narrower while the current flows to the right electrode. Finally, Route 1 becomes dominant in the total junction current. In this point, the right heteroatom is less effective, which will be further demonstrated in the following simulation. This transmission presentation also proposes that the system with $\alpha$-connection is more conductive than TC.

Comparing $\mathrm{O}$ and $\mathrm{S}$ substitutions in the cases of both $\alpha$ - and $\beta$-connected heteroacenes, there is no obvious difference in the spatial presentation of the transmission coefficient, showing that they have the same mechanism in the modulation of electron transfer. On the contrary, a completely different pattern can be observed in $\beta$-connected heteroacenes when compared to $\alpha$-connection. The heteroatoms impede the current flow in Route 1, and we see that there is almost no transmission population around the left heteroatom. In Route 2, the current is observed to decay significantly and there is only a small transmission population on the right heteroatom. Moreover, the current exchange between these two routes is also less efficient. Consequently, the 
total junction conductivity of the $\beta$-connection is rather small.

It is interesting to compare again the role of the positions of the two heteroatoms. When the bias is applied from left to right, the left heteroatom plays a larger role in determining the current than the right one. This conclusion may be instructive for further design of molecular devices such as wires and rectifiers. In order to obtain a comprehensive understanding of the electron transfer details, we will model a series of analogues, in which specific electron transfer routes are blocked.

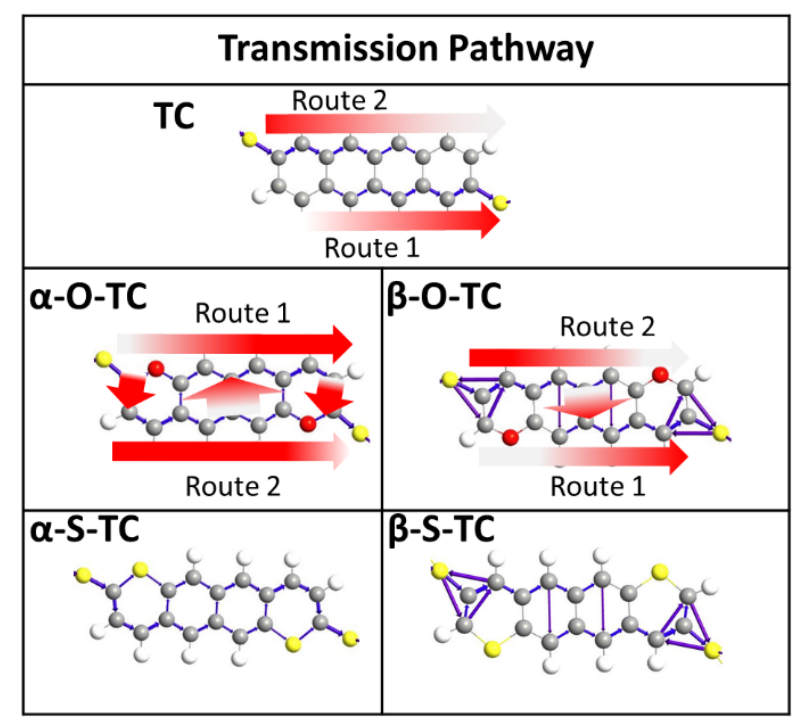

Figure 4. Electron-transport pathways at the Fermi level for all molecules, where the bold red arrows are indicative of the direction and intensity of current transmission.

\section{Investigation on the electron-transfer pathway and QI effect}

All artificial molecules studied in the present section are designed based on $\alpha$-STC, by removing certain sulfur and carbon atoms. When any part of the molecule is removed, hydrogen saturation is employed, and only these hydrogen atoms in the constructed molecules are optimized. These artificial molecules can help us to understand better the role of heteroatoms in electron transport behavior of the sixmembered ring in an $\alpha$-S-TC molecule. The analysis of the spatial distribution of HOMO and LUMO is instructive for understanding the influence of heteroatoms on the junction transport. As shown in Figure 1a, the sulfur atoms make a significant contribution to the well-conjugated HOMO and LUMO distribution, therefore, the removal of these heteroatoms may destroy the electronic conjugated structure. To 
clarify the role of the heteroatom position, we compared $\alpha$-S-TC-1 and $\alpha$-S-TC-2 to $\alpha$ $\mathrm{S}-\mathrm{TC}$ as shown in Figure 5. When the left $\mathrm{S}$ heteroatom, the one close to the positive electrode, is removed, the current becomes smaller in the large bias regime. Specifically, under a bias larger than $0.9 \mathrm{~V}$, the current is reduced to almost the half of the original $\alpha$-S-TC molecule. This result indicates that the left $\mathrm{S}$ heteroatom plays a positive role in the junction transport at large bias. As also seen from Figure 4, after the current injects into the molecule, two pathways are present with one passing through the left $\mathrm{S}$ heteroatom. The current will be increased when two pathways come together constructively. Therefore, the left $\mathrm{S}$ heteroatom should contribute to the junction conductance through constructive QI. When it is removed, the constructive QI disappears and therefore, the current decreases. On the contrary, the right S heteroatom, which is the one close to the negative electrode, plays almost no effect on the junction transport. Both current-bias curves of $\alpha$-S-TC and $\alpha$-S-TC-2 are very close to each other, indicating they follow the same electron transfer manner.

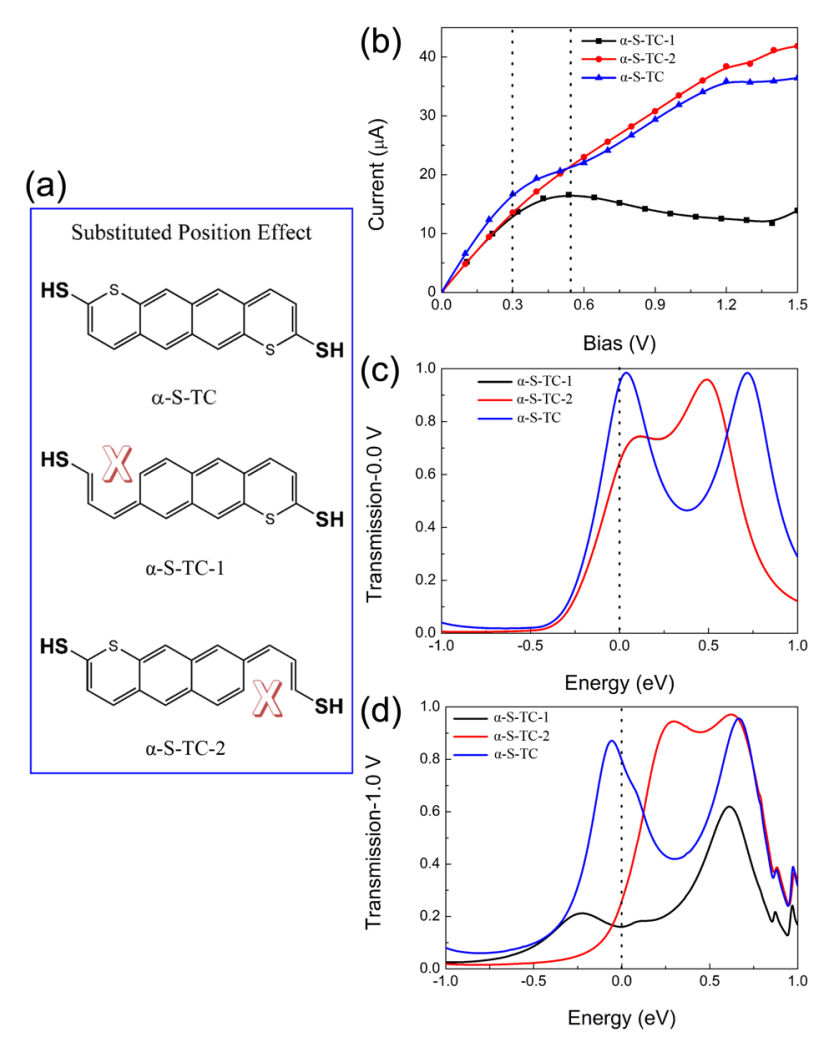

Figure 5 (a) The molecular structures of $\alpha$-S-TC, $\alpha$-S-TC- 1 and $\alpha$-S-TC-2; (b) the plots of $I-V$ curves of these models; (c) the transmission spectra of these molecular junction at zero-bias, where the curves of $\alpha$-S-TC- 1 and $\alpha$-S-TC- 2 are the same; and (d) the transmission spectra at $1.0 \mathrm{~V}$ bias. 
It is important to emphasize that the current values, i.e., the conductivities of $\alpha$-STC- 1 and $\alpha$-S-TC-2, are identical when the bias is less than $0.3 \mathrm{~V}$. This indicates that the heteroatom effect is strongly dependent on bias. It plays a role only when the bias is large, i.e., the electric field across the molecule is strong enough to drive a large current penetrating far into the two electron transfer routes. When the electric field is small, i.e., under small bias, the current is small and the current streaming through the heteroatom is negligible. This is consistent with the data in Figure 2, showing the same current values at a small bias for $\mathrm{O}$ - and S-substitutions when they have the same connection position. This feature is also observed from the zero-bias transmission spectra in Figure 5c, in which two curves merged together for $\alpha$-S-TC-1 and $\alpha$-S-TC2. However, both of these models seem to be less conductive than $\alpha$-S-TC, most likely due to the structural relaxation or modulation of the frontier energy levels by the heteroatoms.

The transmission spectrum at large bias may give insight into the conductance difference between $\alpha$-S-TC- 1 and $\alpha$-S-TC- 2 in Figure $5 \mathrm{~d}$. This spectrum of $\alpha$-S-TC has only a small difference when compared to the zero-bias spectrum. The transmission peak just above the Fermi level of $\alpha$-S-TC-2 shifts positively and increases noticeably at $1.0 \mathrm{~V}$ bias. On the contrary, the transmission peak above the Fermi level of $\alpha$-S-TC1 shifts negatively, below the Fermi level. In addition, the transmission amplitude is much decreased at the same bias. Obviously, the transportation behavior of the two models is dominated by the transmission spectra. From the current-bias curve, we can find that $\alpha$-S-TC-1 may have negative differential resistance, which is most likely caused by the transmission peaks shifting across the Fermi level.

To estimate the current exchange between Route 1 and Route 2, we further compare $\alpha$-S-TC-2, $\alpha$-S-TC-3 and $\alpha$-S-TC-4 as shown in Figure 6 . The length of Route 2 is reduced after removing the carbon atoms from the fused benzene. The current-voltage curves of $\alpha$-S-TC-2 and $\alpha$-S-TC-3 are similar, especially at a bias less than $0.3 \mathrm{~V}$, proving that the last two rings contribute less to the current exchange from Route 2 to Route 1 . However, as shown by the $I-V$ curve of $\alpha$-S-TC-4, when Route 2 is broken at the second ring, the current is reduced to almost half of that of $\alpha$-S-TC-2 and $\alpha$-S-TC3 , indicating the importance of the second ring for the current exchange between the two routes. The results also imply that the first and second rings have a similar contribution to the current exchange. Figure $6 \mathrm{c}$ presents the transmission spectra of the 
three samples at zero-bias. Although the shape of the spectra is different between $\alpha$-STC-2 and $\alpha$-S-TC-3 at energies above the Fermi level, their transmission values at the Fermi level are almost identical, resulting in a similar conductivity at low bias. $\alpha$-STC-3 and $\alpha$-S-TC-4 have a similar shape of the transmission spectrum, but, the absolute value is much reduced for $\alpha$-S-TC-4. This indicates the low conductivity of $\alpha$-S-TC-4 at low bias. Under the higher bias, for example $1.0 \mathrm{~V}$, the transmission spectra of $\alpha$-STC-2 and $\alpha$-S-TC-3 are positively shifted and increased as shown in Figure 6d. In contrast, the transmission wave of $\alpha$-S-TC- 4 has only a positive shift. Therefore, the current of $\alpha$-S-TC-2 and $\alpha$-S-TC-3 keep increasing with bias, while $\alpha$-S-TC-4 stays relatively unchanged over a large bias region.

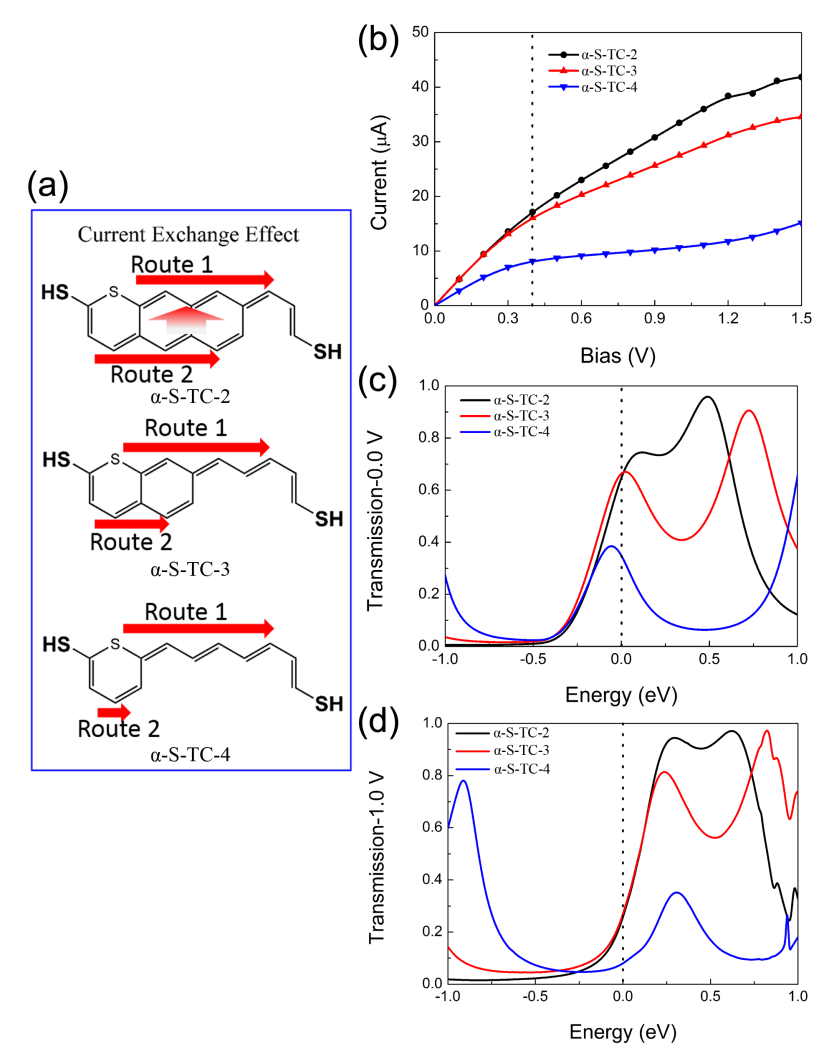

Figure 6 (a) The molecular structures of $\alpha$-S-TC-2, $\alpha$-S-TC-3 and $\alpha$-S-TC-4; (b) The plots of I-V curves of these models; (c) the transmission spectra of these molecular junction at zero-bias; and (d) the transmission spectra at $1.0 \mathrm{~V}$ bias.

As shown in Figures 4 and 5, the heteroatom close the positive electrode appears to be more important than the one on the opposite side. The two heteroatoms play different roles in electron transfer, though they have the symmetric junction structure. This asymmetric dominance is of great importance as it might be used for the design of 
molecular rectifiers. To further clarify the role of the left heteroatom, we compared $\alpha$ S-TC-4, $\alpha$-S-TC-5 to the $\alpha$-S-TC molecule (Figure 7). As discussed above, the left heteroatom plays a positive role through constructive interference, showing an increase in current through $\alpha$-connected heteroacenes relative to TC. From the transmission pathway analysis as given in Figure 4, the particular point of the constructive interference is located at the meta-position to the heteroatom denoted by the red circle. However, it is not clear about the nature of the ortho-position. Comparing $\alpha$-S-TC-4 and $\alpha$-S-TC, we can see that the current exchange is more important when the second ring is unbroken. This indicates that the constructive interference takes place at the meta-position, and the current flowing through Route 2 together the current exchange to Route 1 is the dominant mechanism. When the left heteroatom is removed from $\alpha$ S-TC-4, the quantum interference no longer exists. Therefore, there is only electron transfer pathway through the alternative double and single bonds, which has been demonstrated to be a very efficient electron transfer route ${ }^{40}$. As a result, $\alpha-\mathrm{S}-\mathrm{TC}-4$ has similar conductivity to $\alpha$-S-TC ]. However, they still have minor differences depending on the bias applied. In particular, when the bias is less than $0.7 \mathrm{~V}, \alpha$-S-TC is more conductive than $\alpha$-S-TC-5, indicating a weak constructive interference exists at the meta-position. On the contrary, a slight destructive interference takes place when the bias is larger than $0.7 \mathrm{~V}$. 


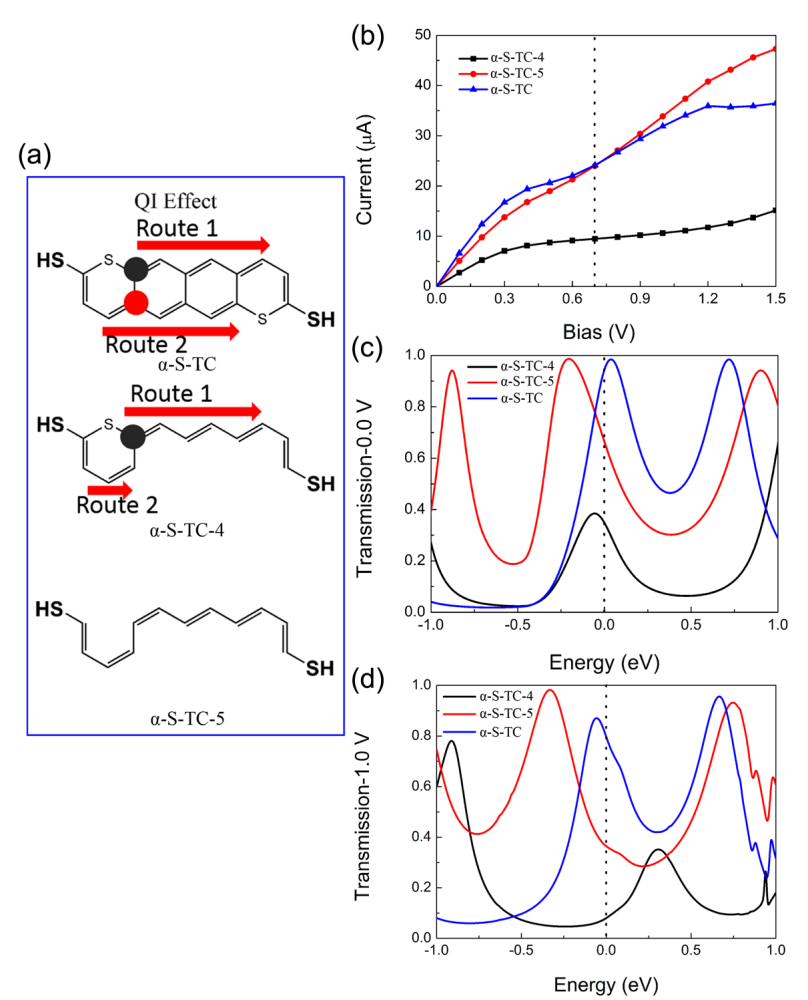

Figure 7 (a) The molecular structures of $\alpha$-S-TC, $\alpha$-S-TC-4 and $\alpha$-S-TC-5; (b) The plots of I-V curves of these models; (c) the transmission spectra of these molecular junction at zero-bias; and (d) the transmission spectra at $1.0 \mathrm{~V}$ bias.

Figure $7 \mathrm{c}$ shows the transmission spectra at zero-bias for these samples. The transmission values at zero-bias follow the order of $\alpha$-S-TC $>\alpha$-S-TC-5 $>\alpha$-S-TC-4, which agrees well with the conductivity as given in Figure $7 \mathrm{~b}$. At $1.0 \mathrm{~V}$ bias, the transmission value of $\alpha$-S-TC is decreased to $90 \%$ of that at zero-bias, but the transmission amplitude is only slightly lower for $\alpha$-S-TC-5. As a result of the integral nature of current in the bias window, the current of $\alpha$-S-TC-5 is even larger than that of $\alpha$-S-TC-4 at bias above $0.7 \mathrm{~V}$. These results demonstrate that the heteroatom plays multiple roles in electron transportation, depending on the bias applied.

\section{Conclusions}

In the present work, we have studied the electron-transport behavior of tetracene (TC) with diene conjugation and heteroacenes (S/O-TC) with quinoidal conjugation. The conductivity of these molecular junctions corresponds to three categories, where $\alpha$-connected heteroacenes have the greatest conductance, followed by TC, and $\beta$ connected S/O-TC has the lowest. These results indicate that the quinoidal conjugation 
with diradical character is more beneficial to electron transport compared to the diene conjugation. Importantly, it has been shown that the connection of anchoring groups can effectively regulate the conductivity of organic molecular junctions. In contrast, the heteroatoms have relatively little influence on the junction conductivity. Additionally, five artificial models ( $\alpha$-S-TC-1 to $\alpha$-S-TC-5) have been designed and used to gain a better understanding of the effect of heteroatoms on electron transport of molecular junction. It has been found that the left S heteroatom of $\alpha$-S-TC is able to contribute to the conductivity of junctions by constructive quantum interference, but not the right $\mathrm{S}$ heteroatoms. Furthermore, two electron transport paths have been identified in heteroacene, between which current exchange exists. The electron transport pathways involving alternative double and single bonds will tend to offer the lowest resistance. The effective electron transfer path could visually reflect the relationship between the electron transfer behavior and molecular structure. The insight gained in the present study on the effective electron-transport pathway helps us to have a better understanding of the electron transfer behavior in the complex heteroacenes system, and to guide the design and screening for future molecular electronic systems with optimized performance.

\section{Acknowledgement}

This work was supported by the Zhejiang Provincial Natural Science Foundation of China [Q18E040005], [2017AY13009] and National Natural Science Foundation of China [51861145202], [51802121].

\section{References}

1. X. Dong, X. Wang, C. Jia, T. Lee and X. Guo, Chemical Reviews, 2016, 116, 4318-4440.

2. R. M. Metzger, Journal of Materials Chemistry, 2015, 115, 5056-5115.

3. L. Sun, Y. A. Diazfernandez, T. A. Gschneidtner, F. Westerlund, S. Laraavila and K. Mothpoulsen, Chemical Society Reviews, 2014, 43, 7378-7411.

4. M. Ratner, Nature Nanotechnology, 2013, 8, 378-381.

5. Cuevas and JuanCarlos, Molecular electronics : an introduction to theory and experiment, World Scientific, 2010.

6. J. Z. Liu, X.; Al-Galiby, Q.; Huang, X.; Zheng, J.; Li, R.; Huang, C.; Yang, Y.; Shi, J.; Manrique, D. Z.; Lambert, C. J.; Bryce, M. R.; Hong, W. , Angew. Chem. Int. Edi. , 2017, 129, 13241-13245.

7. J. H. Haib, I.; Renaud, J. M., Science, 2003, 300, 1384-1389. 
8. X. Yin, Y. Zang, L. Zhu, J. Z. Low, Z. F. Liu, J. Cui, J. B. Neaton, L. Venkataraman and L. M. Campos, Sci Adv, 2017, 3, eaao2615-1-2615-5.

9. C. Jia, A. Migliore, N. Xin, S. Huang, J. Wang, Q. Yang, S. Wang, H. Chen, D. Wang and B. Feng, Science, 2016, 352, 1443-1445.

10. R. M. Metzger, Chemical Reviews, 2003, 34, 3803-3834.

11. J. V. Trasobares, D.; Théron, D.; Clément, N., Nat. Commun., 2016, 7, 12850-1285112850-12859.

12. Michael Galperin, $\dagger$, M. A. R. And, $\uparrow$ and A. Nitzan $\ddagger$, Nano Letters, 2005, 5, 125-130.

13. R. Pati, M. Mcclain and A. Bandyopadhyay, Physical Review Letters, 2008, 100, 2468011-246801-4.

14. J. S. Lindsey and D. F. Bocian, Accounts of Chemical Research, 2011, 44, 638-650.

15. L. Xiang, J. L. Palma, Y. Li, V. Mujica, M. A. Ratner and N. Tao, Nature communications, 2017, 8, 14471-1-14471-10.

16. Y. Komoto, S. Fujii and M. Kiguchi, Materials Chemistry Frontiers, 2017, 2, 214-218.

17. H. Dong, H. Zhu, Q. Meng, X. Gong and W. Hu, Chemical Society Reviews, 2012, 41, 1754-1808.

18. X. Li, L. Cao, H. L. Li, H. Wan and G. Zhou, Journal of Physical Chemistry C, 2016, 120, 3010-3018.

19. G. Ma, L. Sun, R. Zhang, Z. Shen and S. Hou, Chemical Physics, 2010, 375, 67-72.

20. L. B. Zhao, J. L. Chen, M. Zhang, D. Y. Wu and Z. Q. Tian, Journal of Physical Chemistry C, 2015, 119, 4949-4958.

21. R. W. Yan, X. Jin, S. Y. Guan, X. G. Zhang, R. Pang, Z. Q. Tian, D. Y. Wu and B. Mao, Journal of Physical Chemistry C, 2016, 120,11820-11830.

22. G. Kuang, S. Z. Chen, W. Wang, T. Lin, K. Chen, X. Shang, P. N. Liu and N. Lin, Journal of the American Chemical Society, 2016, 138,11140-11143.

23. F. Chen, X. Li, J. Hihath, Z. Huang and N. Tao, Journal of the American Chemical Society, 2006, 128, 15874-15881.

24. M. Wang, Y. Wang, S. Sanvito and S. Hou, Journal of Chemical Physics, 2017, 147, 054702-1-054702-5.

25. J. Z. Liu, X.; Al-Galiby, Q.; Huang, X.; Zheng, J.; Li, R.; Huang, C.; Yang, Y.; Shi, J.; Manrique, D. Z.; Lambert, C. J.; Bryce, M. R.; Hong, W., Angew. Chem. Int. Edi., 2017, 129, 13241-13245.

26. H. L. Dr, Y. Cui, N. Gao and J. Z. Prof, Chemphyschem, 2010, 11, 1895-1902.

27. G. C. Solomon, D. Q. Andrews, T. Hansen, R. H. Goldsmith, M. R. Wasielewski, R. P. Van Duyne and M. A. Ratner, Journal of Chemical Physics, 2008, 129, 966.

28. S. H. Ke, W. Yang and H. U. Baranger, Nano Letters, 2008, 8, 3257-3261.

29. P. Sautet and C. Joachim, Chemical Physics Letters, 1988, 153, 511-516.

30. A. Roi Baer and Daniel Neuhauser, Journal of the American Chemical Society, 2002, 124, 4200-4201.

31. H. B. Weber, J. Reichert, F. Weigend, R. Ochs, D. Beckmann, M. Mayor, R. Ahlrichs and H. V. Löhneysen, Chemical Physics, 2002, 281, 113-125.

32. C. Liu, D. Walter, D. Neuhauser and R. Baer, Journal of the American Chemical Society, 2003, 125, 13936-13937.

33. D. Walter, D. Neuhauser and R. Baer, Chemical Physics, 2004, 299, 139-145. 
34. J. Y. Kim, I. J. Chung, Y. C. Kim and J. W. Yu, Chemical Physics Letters, 2004, 398, 367371.

35. E. Maggio, G. C. Solomon and A. Troisi, Acs Nano, 2014, 8, 409-418.

36. X. Liu, S. Sangtarash, D. Reber, D. Zhang, H. Sadeghi, J. Shi, Z. Y. Xiao, W. Hong, C. J. Lambert and S. X. Liu, Angewandte Chemie, 2016, 56, 173-176.

37. C. M. Guédon, H. Valkenier, T. Markussen, K. S. Thygesen, J. C. Hummelen and S. J. V. D. Molen, Nature Nanotechnology, 2012, 7, 305-309.

38. F. Schwarz, M. Koch, G. Kastlunger, H. Berke, R. Stadler, K. Venkatesan and E. Lã $\uparrow$ Rtscher, Angewandte Chemie International Edition, 2016, 55, 11781-11786.

39. G. Yang, S. Sangtarash, Z. Liu, X. Li, H. Sadeghi, Z. Tan, R. Li, J. Zheng, X. Dong and J. Liu, Chemical Science, 2017, 8, 7505.

40. a) H. Liu, W. Ni, J. Zhao, N. Wang, Y. Guo, T. Taketsugu, M. Kiguchi and K. Murakoshi, Journal of Chemical Physics, 2009, 130, 244501-1-244501-8; b ) H. Liu, Z. Xu, N. Wang, C. Yu, N. Gao, J. Zhao and N. Li, Journal of Chemical Physics, 2010, 132, 244702-1244702-9; c) H. Liu, J. Zhao, F. Boey and H. Zhang, Physical Chemistry Chemical Physics, 2009, 11, 10323-10330; d) H. Liu, H. Wang, J. Zhao and M. Kiguchi, Journal of Computational Chemistry, 2013, 34, 360-365.

41. N. Wang, H. Liu, J. Zhao, Y. Cui, Z. Xu, Y. Ye, M. Kiguchi and K. Murakoshi, J.phys.chem.c, 2009, 113, 7416-7423.

42. Y. He, J. Zhang and J. Zhao, Journal of Physical Chemistry C, 2014, 118, 18325-18333.

43. J. W. Y. Zhao, C.; Wang, N.; Liu, H. M., J. Phys. Chem. C., 2010, 114, 4135-4141.

44. X. Shi and C. Chi, Topics in Current Chemistry, 2017, 375, 68-1-68-39.

45. Q. Ye, J. Chang, X. Shi, G. Dai, W. Zhang, K. W. Huang and C. Chi, Organic Letters, 2014, 16, 3966-3969.

46. S. Dong, T. S. Herng, T. Y. Gopalakrishna, H. Phan, Z. L. Lim, P. Hu, R. D. Webster, J. Ding and C. Chi, Angewandte Chemie International Edition, 2016, 55, 9316-9320.

47. M. J. T. Frisch, G. W.; Schlegel, H. B.; Scuseria, G. E.; Robb, M. A.; Cheeseman, J. R; Scalmani, G.; Barone, V.; Mennucci, B.; Petersson, G. A.; Nakatsuji, H.; Caricato, M.; Li, X.; Hratchian, H. P.; Izmaylov, A. F.; Bloino, J.; Zheng, G.; Sonnenberg, J. L.; Hada, M.; Ehara, M.; Toyota, K.; Fukuda, R.; Hasegawa, J.; Ishida, M.; Nakajima, T.; Honda, Y.; Kitao, O.; Nakai, H.; Vreven, T.; Montgomery, J. A.; Peralta, Jr. J. E.; Ogliaro, F.; Bearpark, M.; Heyd, J. J.; Brothers, E.; Kudin, K. N.; Staroverov, V. N.; Keith, T.; Kobayashi, R.; Normand, J.; Raghavachari, K.; Rendell, A.; Burant, J. C.; Iyengar, S. S.; Tomasi, J.; Cossi, M.; Rega, N.; Millam, J. M.; Klene, M.; Knox, J. E.; Cross, J. B.; Bakken, V.; Adamo, C.; Jaramillo, J.; Gomperts, R.; Stratmann, R. E.; Yazyev, O.; Austin, A. J.; Cammi, R.; Pomelli, C.; Ochterski, J. W.; Martin, R. L.; Morokuma, K.; Zakrzewski, V. G.; Voth, G. A.; Salvador, P.; Dannenberg, J. J.; Dapprich, S.; Daniels, A. D.; Farkas, O.; Foresman, J. B.; Ortiz, J. V.; Cioslowski, J.; and Fox, D. J.Gaussian, Inc, Wallingford CT, 2013. Gaussian 09, Revision E. 01.

48. Y. Zhao and D. G. Truhlar, Journal of Physical Chemistry A, 2006, 110, 13126-13130.

49. I. Y. Zhang, J. Wu and X. Xu, Chemical Communications, 2010, 46, 3057-3070.

50. Z. Cai, W. Y. Lo, T. Zheng, L. Li, N. Zhang, Y. Hu and L. Yu, Journal of the American Chemical Society, 2016, 138, 10630-10635.

51. M. F. Kiguchi, S., B. Chem. Soc. Jpn, 2016, 90, DOI: 10.1246/bcsj.20160229. 
52. Atomistix ToolKit; QuantumWise A/S, http://www.quantumwise.com.

53. J. E. Norton and J. L. Brédas, Journal of the American Chemical Society, 2008, 130, 1237712384.

54. J. E. Anthony, Chemical Reviews, 2007, 38, 5028-5048.

55. John, \#x and E. A. Prof, Angewandte Chemie International Edition, 2008, 47, 452-483.

56. Z. Sun, Q. Ye, C. Chi and J. Wu, Chemical Society Reviews, 2013, 44, 7857-7889.

57. X. W. Gao, L.; Ohtsuka, Y.; Jiang, D.; Zhao, Y.; Nagase, S.; Chen, Z., J. Am. Chem. Soc. , 2009, 131, 9663-9669.

58. M. K. Jeffries-El, B. M.; Hale, B. J., Macromolecules., 2014, 47, 7253-7271.

59. B. Kim, S. H. Choi, X. Y. Zhu and C. D. Frisbie, Journal of the American Chemical Society, 2011, 133, 19864-19877.

60. T. S. Markussen, R.; Thygesen, K. S., Nano Lett, 2010, 10, 4260-4265.

61. Y. H. Jang, S. Hwang, Y. H. Kim and S. S. Jang, Journal of the American Chemical Society, 2004, 126, 12636-12645.

62. H. J. Yun and W. A. G. Iii, J.phys.chem.c, 2010, 114, 4646-4651.

63. S. Datta, Physics Today, 1996, 49, 70-70.

64. Y. Meir and N. S. Wingreen, Physical Review Letters, 1992, 68, 2512-2515.

65. G. C. Solomon, C. Herrmann, T. Hansen, V. Mujica and M. A. Ratner, Nature Chemistry, 2010, 2, 223-228. 